\title{
Olfactory neuron turnover in adult Drosophila
}

\section{Ismael Fernández-Hernández ${ }^{1^{*}}$, Eric Hu${ }^{1}$, Michael A. Bonaguidi ${ }^{12^{*}}$}

$2 \quad{ }^{1}$ Department of Stem Cell Biology and Regenerative Medicine, Eli and Edythe Broad Center for

3 Regenerative Medicine and Stem Cell Research, Keck School of Medicine, University of Southern

4 California, Los Angeles, CA, USA

$5 \quad{ }^{2}$ Department of Biomedical Engineering, Viterbi School of Engineering, University of Southern

6 California, Los Angeles, CA, USA; Department of Gerontology, Davis School of Gerontology,

7 University of Southern California, Los Angeles, CA, 90089, USA; Zilkha Neurogenetic Institute,

8 Keck School of Medicine, University of Southern California, Los Angeles, CA, USA

$9 *$ Correspondence:

10 Ismael Fernández-Hernández, $\mathrm{PhD}$

11 ismaelfe@usc.edu

12 Michael A. Bonaguidi, PhD

13 mbonagui@usc.edu

14 Keywords: Olfactory neuron, adult neurogenesis, apoptosis, turnover, lineage tracing, 15 circuitry, Drosophila.

\section{ABSTRACT}

17 Sustained neurogenesis occurs in the olfactory epithelium of several species, including humans, to 18 support olfactory function throughout life. We recently developed a modified lineage tracing method 19 to identify adult neurogenesis in Drosophila. By applying this technique, here we report on the 20 continuous generation of Olfactory Sensory Neurons (OSN) in the antennae of adult Drosophila. New 21 neurons develop sensory dendrites and project axons targeting diverse glomeruli of the antennal lobes 22 in the brain. Furthermore, we identified sustained apoptosis of OSN in the antennae of adult flies, 23 revealing unexpected turnover in the adult olfactory system. Our results substantiate Drosophila as a 24 compelling platform to expedite research about mechanisms and compounds promoting neuronal regeneration, circuit remodeling and its contribution to behavior in the adult. 
Sensory systems mediate the processing of external cues for the establishment of environmental and inter-individual interactions. Olfaction is essential in most species to identify food and mates, to escape from predators, and to establish memories. In order to sustain these functions throughout life, adult neurogenesis in the olfactory neuroepithelium occurs across several species, including humans (Bayramli et al., 2017; Durante et al., 2020; Fletcher et al., 2017; Graziadei and Graziadei, 1979; Graziadei and Okano, 1979; Weiler and Farbman, 1997).

Research on the olfactory system of the fruit fly Drosophila melanogaster has provided detailed insight on the mechanisms mediating odor coding and its evoked behaviors and memory formation at the molecular, cellular and circuitry level (Busto et al., 2010; Cognigni et al., 2018; Masse et al., 2009). Yet, generation of Olfactory Sensory Neurons (OSN) in the adult fly has not been reported thus far. Its identification would expedite a detailed analysis of the programs controlling OSN proliferation in response to environmental toxins, adult circuit remodeling, and the contribution of adult-born neurons to modulate odor-evoked behavior and memory.

The cellular architecture and function of the adult olfactory system in Drosophila is remarkably conserved to that in vertebrates, but numerically simpler. About 1300 OSN are clustered into sensory hairs called sensilla. These reside on the surface of the third antennal segment and the maxillary palps of flies, the olfactory organs equivalent to vertebrate's olfactory epithelium. Here, most OSN express one of $\sim 60$ Olfactory Receptors (OR), as well as Orco, a related co-receptor. The axons of OSN project into diverse glomeruli in the Antennal Lobes (AL) in the brain, the counterparts to vertebrate's olfactory bulb. Local Interneurons (LI) innervate the AL to mediate intra- and inter-glomerular inhibition. Finally, Projections Neurons (PN) convey the olfactory signals to the Mushroom Bodies (MB) and Lateral Horn (LH), the centers for integration and storage of olfactory information, equivalent to the olfactory cortex and higher processing centers in vertebrates (Galizia and Sachse, 2009; Hallem and Carlson, 2004; Masse et al., 2009; Vosshall and Laissue, 2008). Extensive genetic

51 tools available in Drosophila grant multilevel analysis of the olfactory circuit in the adult, in a shorter time and at a lower cost than current vertebrate models.

53 We recently developed a modified lineage tracing system to detect generation of neurons in the optic 54 lobes and the mechanosensory systemin the antennae of adult flies (Fernández-Hernández et al., 2019). 55 We therefore hypothesized that the olfactory system also retains this capacity. By implementing this 
method, we identified sustained generation of OSN over three weeks in the antennae of adult Drosophila. New OSN develop sensory dendrites and project axons to the antennal lobes in the brain. Furthermore, we detected continuous apoptosis of OSN in the antennae, thus revealing turnover in the olfactory system of adult flies. Our results substantiate Drosophila as an advantageous platform to analyze and promote neuronal plasticity in the adult olfactory system at different levels.

\section{RESULTS}

\section{P-MARCM detects adult-born OSN in Drosophila}

We recently developed P-MARCM, a permanently-active lineage tracing system to label mitoticallyderived cells in adult Drosophila tissues, including those appearing at low frequency, in a cell typespecific manner (Fernández-Hernández et al., 2019). Briefly, in this method, sustained expression of the recombinase FLP triggered by a heat shock (HS) mediates recombination of homologous chromosomes at G2 phase, allowing expression of cytoplasmic GFP and nuclear RFP in hemiclones of daughter cells upon mitosis. Specificity for labelling in the lineage is achieved by incorporation of cell type-specific GAL4 lines (Figure 1A-C). P-MARCM successfully detected adult neurogenesis and regeneration in the optic lobes and Johnston's Organ (Fernández-Hernández et al., 2013, 2019). Because adult neurogenesis occurs in the visual and auditory/vestibular sensory systems, we hypothesized that the adult olfactory system also retains this capacity. To investigate this, we applied P-MARCM with the pan-neuronal line $n s y b-G A L 4$ (P-MARCM nsyb), in order to maximize the number of new OSN detected in the antennae (Vosshall et al., 2000; Sethi and Wang, 2017). We activated P-MARCM nsyb on 3-5 days-old female flies by heat shock and quantified OSN neurogenesis from confocal images of antennae dissected weekly over the next 3 weeks (Figure 1D,E). Remarkably, this approach captured OSN generated over time throughout the third antennal segment (Figure 1F). Indeed, while background labeling in non-heat-shocked flies ( 0 weeks, $0 \mathrm{w})$ was only 4.0 +/-0.5 SEM OSN/antenna ( $\mathrm{n}=27)$, we identified 9.7 +/- 2.7 SEM OSN/antenna at 1w ( $\mathrm{n}=15) ; 18.4$ +/1.7 SEM OSN/antenna at $2 \mathrm{w}(\mathrm{n}=21)$, and 41.0 +/-2.8 SEM OSN/antenna at 3w $(\mathrm{n}=14)$ (Figure 1G). Clustering antennae by the number of OSN labeled reveals continuous neurogenesis over time (Figure $1 \mathrm{H})$. This is also demonstrated by an increase in the number of antennae with OSN labeled beyond background levels over time (i.e. $\geq 10$ OSN/antenna, or mean +2 sd $(4.0+2 * 2.5)=9$ OSN/antennae) 4 (Figure 1I). Altogether, these results reveal unexpected proliferative potential in the adult Drosophila 8 olfactory system. 


\section{Adult-born OSN develop dendrites and target brain circuitry}

Next, we evaluated the cellular features of adult-born OSN. Firstly, detailed analysis of confocal images revealed that new OSN develop sensory dendrites, which are essential for olfaction (Figure 2A). Indeed, here the olfactory receptors are recruited to bind volatile small molecules from the environment to trigger olfaction (Larsson et al., 2004). Secondly, new OSN extend axons, which navigate through the second antennal segment towards the central brain (Figure 2B). Finally, axons of new OSN target diverse glomeruli of the Antennal Lobes (AL) in the central brain (Figure 2C). We consistently identified these features in all samples analyzed. Taken together, the cellular and circuitry features identified in new OSN strongly suggest that they mature and have the potential to functionally remodel the adult olfactory system.

\section{Different types of OSN are generated in the adult antenna}

We next evaluated whether one or more types of OSN are generated in the antenna, based on different cellular and circuit features. Firstly, the diversity of OSN in the antenna is harbored in four types of sensilla: trichoid, basiconic, intermediate, and coeloconic, which segregate into different domains on the third antennal segment (Shanbhag et al., 1999; Couto et al., 2005; Grabe et al., 2016) (Figure 3A). By applying P-MARCM, we identified new OSN throughout the third antennal segment spanning the domains of the different types of sensilla, indicating that multiple types of OSN are generated (Figure 3B). Second, OSN can also be linked to one type of sensillum by the length of their dendrites (Figure 3C). At this level, we identified OSN with long and short dendrites, characteristic of trichoid and coeloconic or small basiconic sensilla, respectively (Shanbhag et al., 1999) (Figure 3D). Third, at the circuit level, OSN expressing the same OR converge to a single glomerulus in the AL (Couto et al., 2005; Fishilevich and Vosshall, 2005; Vosshall et al., 2000) (Figure 3E). Here, we observed different glomeruli in the AL innervated by the adult-born OSN, indicating that more than one class of OSN were generated (Figure 3F). These representative features were consistently observed across the different samples analyzed. Taken together, these observations indicate that adult neurogenesis is not restricted to a single type of olfactory neurons (i.e. those expressing a single combination of olfactory receptors), but it rather spans to neurons of different subtypes.

\section{Turnover of adult OSN}

We next asked whether new OSN detected by P-MARCM might replace lost ones in an ongoing turnover process, as it’s been suggested for the Johnston's Organ (Fernández-Hernández et al., 2019). 
116 To answer this, we expressed the genetically-encoded reporter of apoptosis $U A S$-GC3Ai (GFP sensor 117 Caspase-3-like protease Activity indicator (Schott et al., 2017)) with the pan-neuronal nsyb-GAL4 118 driver. Briefly, expression of GC3Ai produces a non-fluorescent GFP due to an intervening caspase119 recognizing sequence; upon cleavage of this sequence by active caspases, GFP becomes rapidly 120 fluorescent in apoptotic cells (Figure 4A). By using this method, we detected GFP expression in OSN 121 with condensed nuclei, a hallmark of apoptosis, thus validating the utility of GC3Ai as an apoptosis 122 reporter in the Drosophila olfactory system (Figure 4B). Indeed, GC3Ai revealed sustained apoptosis 123 of OSN in the antennae of female and male flies over three weeks (Figure 4C), with no significant 124 difference between sexes at each time point analyzed (7.0+/-0.6 SEM apoptotic OSN/antenna at $1 \mathrm{w}$, $125 \mathrm{n}=31$ total antennae; 9.5+/- 0.5 SEM at $2 \mathrm{w}, \mathrm{n}=47 ; 8.1+/-0.6$ SEM at $3 \mathrm{w}, \mathrm{n}=23$; Figure 4D). The 126 consistent identification of apoptotic cells at discrete timepoints strongly suggest that OSN are 127 continuously eliminated over the analysis period in the adult.

128 In order to correlate OSN apoptosis with proliferation, we focused on data from female flies, since P129 MARCM relies on X chromosome mitotic recombination and labelling, and is therefore active only in 130 females. Statistical analysis reveals a significant increase in OSN apoptosis in 2w-old female flies (10.1 $131+/-1.0$ SEM OSN/antenna, $\mathrm{n}=15)$ with respect to $1 \mathrm{w}$-old flies $(7.0+/-0.8$ SEM OSN/antenna, $\mathrm{n}=22$; $132 p=0.04$, one-way ANOVA, Figure 4E). This correlates with a compensatory, significant increase in 133 OSN proliferation from $2 \mathrm{w}(19.2+/-2.1$ SEM OSN/antenna, $\mathrm{n}=17)$ to $3 \mathrm{w}(41.0+/-2.8$ SEM $134 \mathrm{OSN} /$ antenna, $\mathrm{n}=14 ; p=0.04$, Kruskal-Wallis test, Figure 4F). Taken together, the sustained levels of 135 apoptosis detected by GC3Ai and the continuous proliferation captured by P-MARCM over three 136 weeks indicate turnover of OSN, revealing unexpected cellular plasticity in the adult Drosophila 137 olfactory system.

\section{Adult neurogenesis across sensory systems in Drosophila}

139 Finally, we compared adult neurogenesis in the olfactory system with that occurring in other sensory 140 systems. New neurons have also been detected by P-MARCM in the visual and auditory/vestibular 141 system of 3 week-old adult flies (Fernández-Hernández et al., 2019) (Supplementary Figure 1A-C). A 142 comparative analysis across the 3 sensory systems reveals the highest rate of neurogenesis in the 143 olfactory system of the third antennal segment (41.0+/-2.8 SEM OSN/antenna, $\sim 3 \%$ of $\sim 1300$ total 144 OSN per antenna, $100 \%$ antennae $(n=14)$, this work), followed by the mechanosensory 145 auditory/vestibular JO neurons in the second antennal segment (11.2 JO neurons/antenna, $2.2 \%$ of $146 \sim 500$ JO neurons per antenna, interpolation for 3 weeks from 2 weeks $(n=7)$ and 4 weeks $(n=7)$ 
timepoints, 50\% of antennae analyzed), and lastly by the projection neurons in the medulla of the optic lobes 31.0+/- 2.4 SEM neurons per optic lobe, $\sim 0.1 \%$ of $\sim 40,000$ interneurons, $100 \%$ optic lobes analyzed (n=12), (Fernández-Hernández et al., 2019) (Supplementary Figure 1D). These differences in proliferation may reflect different levels of cell death on each system. Indeed, while projection neurons in the optic lobes seem to be much more protected from external damage, the OSN in the antennae are permanently exposed to potential environmental insults which might ultimately lead to their elimination. Yet, the neurogenesis identified reflects a regenerative capacity evolved on each sensory system to repair and potentially restore their functions.

\section{5}

156

157

158

159

160

161

162

163

164

165

166

167

168

169

170

171

172

173

174

175

176

177

\section{DISCUSSION}

Cellular plasticity occurs in defined regions of the adult nervous system. Continuous neurogenesis in the olfactory neuroepithelium supports olfactory function in different species. By implementing the PMARCM lineage tracing method with the pan-neuronal nsby-GAL4 line, here we report sustained generation of OSN over 3 weeks in the antennae of adult Drosophila (Figure 1). This is revealed by an increase in i) the number of OSN generated per antenna, and ii) the number of antennae undergoing OSN neurogenesis over time. We identified adult neurogenesis in 100\% of the antennae analyzed at 3 weeks ( $n=14$ ), with a mean of 41.0 +/-2.8 SEM new OSN/antenna, which represents $\sim 3 \%$ turnover of the $\sim 1300$ OSN in each antenna. Importantly, because P-MARCM labels only hemi-clones from cells in which the system gets activated upon heat-shock, the actual amount of new OSN might be higher. Furthermore, a detailed analysis of P-MARCM images revealed that new OSN develop sensory dendrites and project axons to the AL (Figure 2). These cellular features strongly suggest a complete maturation and integration of new OSN into pre-existing circuitry.

Three lines of evidence suggest that more than one type of OSN are generated in the antennae, by comparing cellular features of new OSN against reference maps (Vosshall et al., 2000; Couto et al., 2005; Grabe et al., 2016): (i) at the topological level, new OSN are scattered throughout the domains of the different sensilla in the third antennal segment; (ii) at the cellular level, new OSN develop dendrites of different lengths: long for Trichoids and short for Coeloconic or small Basiconinc; (iii) at circuitry level, axons of new OSN target different glomeruli in the antennal lobes (Figure 3). An unambiguous assessment of the OSN subtypes generated can be achieved by replacing $n s b y-G A L 4$ in P-MARCM by Olfactory Receptor-specific GAL4 drivers (Vosshall et al., 2000; Couto et al., 2005; Fishilevich and Vosshall, 2005; Lin and Potter, 2015). Yet, our results indicate that neurogenesis spans to different OSN types in the antennae of adult flies over 3 weeks. 
178 We also identified sustained apoptosis of OSN in physiologic conditions, revealed by the genetically179 encoded GC3Ai reporter (Figure 4). Indeed, apoptotic OSN were captured in the antennae of male and 180 female flies over 3 weeks at comparable extents. This cell death might be a consequence of ageing or 181 the accumulation of eventual insults from the environment. Drosophila, as many other animals, 182 strongly rely on olfaction for critical responses and behaviors, such as selection of food, escape from 183 predators and selection of partners for mating. Thus, a compensatory proliferation following 184 elimination of OSN should be in place to preserve these critical functions. Our results showing apoptosis and generation of OSN over time support an ongoing turnover in the olfactory system of adult flies. Whether OSN regenerate following injury, as it occurs in vertebrates (Graziadei et al., 1978; McM Carr and Farbman, 1992; Schwob et al., 1995; Frontera et al., 2016; Cervino et al., 2017), and how ageing affects this capacity, are questions that can be addressed in future experiments using the platform presented here. Furthermore, this platform will facilitate and expedite the screening of molecules promoting proliferation and integration of neurons in an entire adult circuit (FernándezHernández et al., 2016).

While P-MARCM specifically labels adult-born OSN, it does not identify their cell-of-origin. Lowrate self-division of mechanosensory neurons has been recently proposed as a mechanisms driving regeneration in the adult Johnston's Organ (Fernández-Hernández et al., 2019). Whether this mechanism also operates in the olfactory system, or other cell types act as progenitors, can be addressed by combining cell type-specific lineage tracing systems with transcriptomic analysis (Fletcher et al., 2017; Li et al., 2020).

What is the functional role of new OSN? In vertebrates, adult-born olfactory neurons mature and innervate the olfactory bulb to support olfaction (Schwob, 2002)(Blanco-Hernández et al., 2012)(Hurtt et al., 1988)(Ducray et al., 2002). Although we did not test the function of new OSN here, we observed that they develop sensory dendrites and innervate diverse glomeruli in the antennal lobes in the brain, strongly suggesting that they mature, integrate, and remodel the olfactory circuit to sustain its function. Inhibition of programmed cell death in otherwise eliminated OSN during development allows their functional integration in the adult (Prieto-Godino et al., 2020), demonstrating a high degree of plasticity in the olfactory circuit. This capacity further supports the notion that adult-born OSN might be functionally integrated to modulate olfaction. Furthermore, the apparent diversity of OSN generated suggest their potential to respond to multiple odorant molecules, and ultimately to modulate olfactory- 
209 versatility of P-MARCM with the multiple genetic tools available in Drosophila. For instance, the

210 functional integration of newborn OSN can be assessed by circuitry-mapping of axons innervating the

211 AL (Laissue et al., 1999)(Couto et al., 2005)(Grabe et al., 2016), in conjunction with Trans-TANGO

212 (Talay et al., 2017), nsyb-GRASP (Macpherson et al., 2015), and calcium indicators (Chen et al., 2013;

213 Dana et al., 2019) tools. Similarly, their behavioral contribution can be tested by applying established

214 protocols upon selective activation of adult-born OSN by optogenetic tools (Klapoetke et al., 2014), or

215 their genetic silencing, either in a constitutive manner, by expressing TeTx (Sweeney et al., 1995), or

216 in a reversible manner, by expression of $\mathrm{shi}^{\text {ts }}$ (Kitamoto, 2001).

217 In summary, our results demonstrate unexpected plasticity in the olfactory system of adult Drosophila,

218 and establish a unique platform to expedite the identification of molecular mechanisms and the in vivo 219 screening for compounds promoting proliferation, circuit integration and the functional contribution of 220 new neurons in the adult.

\section{MATERIALS AND METHODS}

\section{Fly lines and experimental conditions}

The P-MARCM system has been previously described (Fernández-Hernández et al., 2019). Flies of final genotype hs-Flp,tub-GAL80,neoFRT19A / tub FRT STOP FRT lexA,neoFRT19A ; 20UAS6GFPmyr,UAS-RedStinger / + ; nsyb-GAL4 / 8lexAOp-Flp were used to assess proliferation of OSN.

228 The parental cross was set and kept at $17^{\circ} \mathrm{C}$ during development to minimize FLP induction. Female

229 flies 2-5 days old were picked and blindly assigned to either control group for dissection, or to 230 experimental groups for heat shock at $38^{\circ} \mathrm{C}$ (Ohlstein and Spradling, 2006) for 45 minutes, twice on 231 the same day $\sim 2$ hours apart. Flies were then kept at $25^{\circ} \mathrm{C}$. Antennae and brains were dissected weekly 232 afterwards over 3 weeks.

\section{For GC3Ai experiments}

234 We crossed female virgins of the apoptotic reporter UAS-GC3Ai (Schott et al., 2017) to nsyb-GAL4 235 males. Parental cross and progeny were kept at $25^{\circ} \mathrm{C}$. Antennae of males and females of final genotype 
$236 w ;+; n s y b-G A L 4 / U A S-G C 3 A i$ were dissected at 1, 2 and 3 weeks after eclossion for confocal

237 imaging as described below.

238 Dissection and immunostaining.

239 Antennae were dissected, attached to their corresponding brains in chilled Schneider's medium and 240 then fixed in 3.7\% formaldehyde solution for $20 \mathrm{~min}$. They were then washed with PBS + Triton (1\%) 241 solution for $20 \mathrm{~min}$, followed by a final wash in 1XPBS before incubation with primary nc-82 antibody 242 overnight at $4^{\circ} \mathrm{C}$, followed by incubation with secondary antibody 4 hours at room temperature or 243 overnight at $4^{\circ} \mathrm{C}$. Antibodies used were mouse anti-nc82 (1:10, DSHB) and secondary antibody anti244 mouse Cy5 (1:100, Jackson laboratories). No antibodies were used for GFP and RedStinger fluorescent 245 proteins. Antennae and brains were mounted in Vectashield media with DAPI (Vector laboratories). 246 For mounting, we used double-side sticker spacers (EMS, 70327-9S) to preserve morphology as much 247 as possible (one for antennae, two for brains).

\section{Statistical analysis}

249 Plots and statistical analysis were done using GraphPad Prism, applying Kruskal-Wallis test for 250 multiple comparisons in Figure $1 \mathrm{G}$ and 4F, unpaired t-Test for data on Figure 4D and one-way 251 ANOVA for data on Figure 4E. Error bars represent SEM in all plots. SEM on experiments with binary 252 outcomes (Figure 1I) was calculated as $\sqrt{p(1-p) / n}$, where $\mathrm{p}$ is the frequency of OSN neurogenesis in 253 the analyzed group and $n$ the number of flies considered. For clustering of antennae based on the 254 number of OSN labeled (Figure 1H), any antennae with a given number of OSN higher than the mean $255+2 \mathrm{sd}$ in the control non-heat-shocked group (i.e. background) was regarded as adult neurogenesis. 256 This yielded a threshold of 9.0 OSN/antenna, thus establishing the first cluster as those antennae with 257 0-9 OSN neurons as background and any values above this as adult neurogenesis.

\section{ACKNOWLEDGEMENTS}

260 We thank members of the Bonaguidi Lab for support; Bloomington Drosophila Stock Center (NIH 261 P40OD018537) for UAS-GC3Ai and $n s y b-G A L 4$ lines used in this study; and Developmental Studies Hybridoma Bank, created by the NICHD of the NIH for nc-82 antibodies. 
265 IFH designed and conducted experiments and carried out data collection and analysis. EH conducted

266 experiments. MB supervised experiments, provided funding and resources, and provided input for the 267 manuscript. IFH wrote the manuscript with input from all authors.

\section{FUNDING}

269 Authors acknowledge financial support from USC-CONACYT (Consejo Nacional de Ciencia y 270 Tecnología) Postdoctoral Scholars Program Fellowship and USC Provost's Postdoctoral Scholar 271 Research Grant to I.F.-H.; and National Institutes of Health (R00NS089013, R56AG064077), L.K. 272 Whittier Foundation, Donald E. and Delia B. Baxter Foundation, and Eli and Edythe Broad Foundation 273 grants to M.A.B.

\section{CONFLICT OF INTEREST}

275 The authors declare that the research was conducted in the absence of any commercial or financial 276 relationships that could be construed as a potential conflict of interest. 


\section{REFERENCES}

291 Blanco-Hernández, E., Valle-Leija, P., Zomosa-Signoret, V., Drucker-Colín, R., and Vidaltamayo, R. (2012). Odor Memory Stability after Reinnervation of the Olfactory Bulb. PLoS One 7. doi:10.1371/journal.pone.0046338.

Cervino, A. S., Paz, D. A., and Frontera, J. L. (2017). Neuronal degeneration and regeneration induced by axotomy in the olfactory epithelium of Xenopus laevis. Dev. Neurobiol. 77, 1308-1320. doi:10.1002/dneu.22513.

297 Chen, T.-W., Wardill, T. J., Sun, Y., Pulver, S. R., Renninger, S. L., Baohan, A., et al. (2013). Ultrasensitive fluorescent proteins for imaging neuronal activity. Nature 499, 295-300. doi:10.1038/nature12354.

Couto, A., Alenius, M., and Dickson, B. J. (2005). Molecular, anatomical, and functional organization of the Drosophila olfactory system. Curr. Biol. 15, 1535-1547. doi:10.1016/j.cub.2005.07.034. Nat. Methods 16, 649-657. doi:10.1038/s41592-019-0435-6. 1907-1917. doi:10.1046/j.1460-9568.2002.02044.x. Rep. 3, 1857-1865. doi:10.1016/j.celrep.2013.05.034.

313 Fernández-Hernández, I., Scheenaard, E., Pollarolo, G., and Gonzalez, C. (2016). The translational 314 relevance of Drosophila in drug discovery . EMBO Rep. 17, 471-472.

315 doi:10.15252/embr.201642080.

316 Fishilevich, E., and Vosshall, L. B. (2005). Genetic and functional subdivision of the Drosophila 317 antennal lobe. Curr. Biol. 15, 1548-1553. doi:10.1016/j.cub.2005.07.066.

318 Fletcher, R. B., Das, D., Gadye, L., Street, K. N., Baudhuin, A., Wagner, A., et al. (2017).

319 Deconstructing Olfactory Stem Cell Trajectories at Single-Cell Resolution. Cell Stem Cell 20, 817320 830.e8. doi:10.1016/j.stem.2017.04.003.

321 Frontera, J. L., Raices, M., Cervino, A. S., Pozzi, A. G., and Paz, D. A. (2016). Neural regeneration 322 dynamics of Xenopus laevis olfactory epithelium after zinc sulfate-induced damage. J. Chem. Neuroanat. 77, 1-9. doi:10.1016/j.jchemneu.2016.02.003.

Grabe, V., Baschwitz, A., Dweck, H. K. M., Lavista-llanos, S., Hansson, B. S., and Sachse, S. (2016). Elucidating the Neuronal Architecture of Olfactory Glomeruli in the Drosophila Antennal Lobe. CellReports 16, 3401-3413. doi:10.1016/j.celrep.2016.08.063. 
Graziadei, P. P. C., Levine, R. R., and Graziadei, G. A. M. (1978). Regeneration of olfactory axons and synapse formation in the forebrain after bulbectomy in neonatal-mice (neuroplasticity/synaptogenesis/olfactory glomeruli/neurogenesis/telencephalon).

Hurtt, M. E., Thomas, D. A., Working, P. K., Monticello, T. M., and Morgan, K. T. (1988). Degeneration and regeneration of the olfactory epithelium following inhalation exposure to methyl bromide: Pathology, cell kinetics, and olfactory function. Toxicol. Appl. Pharmacol. 94, 311-328. doi:10.1016/0041-008X(88)90273-6.

Kitamoto, T. (2001). Conditional modification of behavior in drosophila by targeted expression of a temperature-sensitive shibire allele in defined neurons. J. Neurobiol. 47, 81-92. doi:10.1002/neu.1018.

Klapoetke, N. C., Murata, Y., Kim, S. S., Pulver, S. R., Birdsey-Benson, A., Cho, Y. K., et al. (2014). Independent optical excitation of distinct neural populations. Nat. Methods 11, 338-346. doi:10.1038/nmeth.2836.

Laissue, P. P., Reiter, C., Hiesinger, P. R., Halter, S., Fischbach, K. F., and Stocker, R. F. (1999). Three-dimensional reconstruction of the antennal lobe in Drosophila melanogaster. J. Comp. Neurol. 405, 543-552. doi:10.1002/(SICI)1096-9861(19990322)405:4<543::AID-CNE7>3.0.CO;2-A.

Larsson, M. C., Domingos, A. I., Jones, W. D., Chiappe, M. E., Amrein, H., and Vosshall, L. B. (2004). Or83b encodes a broadly expressed odorant receptor essential for Drosophila olfaction. Neuron 43, 703-714. doi:10.1016/j.neuron.2004.08.019.

Li, H., Li, T., Horns, F., Li, J., Xie, Q., Xu, C., et al. (2020). Single-Cell Transcriptomes Reveal Diverse Regulatory Strategies for Olfactory Receptor Expression and Axon Targeting. Curr. Biol. 30, 1189-1198.e5. doi:10.1016/j.cub.2020.01.049.

Lin, C.-C., and Potter, C. J. (2015). Re-Classification of Drosophila melanogaster Trichoid and Intermediate Sensilla Using Fluorescence-Guided Single Sensillum Recording. PLoS One 10, e0139675. doi:10.1371/journal.pone.0139675.

Macpherson, L. J., Zaharieva, E. E., Kearney, P. J., Alpert, M. H., Lin, T. Y., Turan, Z., et al. (2015). Dynamic labelling of neural connections in multiple colours by trans-synaptic fluorescence complementation. Nat. Commun. 6, 1-9. doi:10.1038/ncomms 10024.

McM Carr, V., and Farbman, A. I. (1992). Ablation of the Olfactory Bulb Up-Regulates the Rate of Neurogenesis and Induces Precocious Cell Death in Olfactory Epithelium'.

Ohlstein, B., and Spradling, A. (2006). The adult Drosophila posterior midgut is maintained by pluripotent stem cells. Nature 439, 470-474. doi:10.1038/nature04333.

Prieto-Godino, L. L., Silbering, A. F., Khallaf, M. A., Cruchet, S., Bojkowska, K., Pradervand, S., et al. (2020). Functional integration of "undead" neurons in the olfactory system. Sci. Adv. 6. doi:10.1126/sciadv.aaz7238.

Schott, S., Ambrosini, A., Barbaste, A., Benassayag, C., Gracia, M., Proag, A., et al. (2017). A fluorescent toolkit for spatiotemporal tracking of apoptotic cells in living Drosophila tissues. Dev. 144, 3840-3846. doi:10.1242/dev.149807. 
365 Schwob, J. E. (2002). Neural regeneration and the peripheral olfactory system. Anat. Rec. 269, 33366 49. doi:10.1002/ar.10047.

367 Schwob, J. E., Youngentob, S. L., and Mezza, R. C. (1995). Reconstitution of the rat olfactory 368 epithelium after methyl bromide-induced lesion. J. Comp. Neurol. 359, 15-37.

369 doi:10.1002/cne.903590103.

370 Sethi, S., and Wang, J. W. (2017). A versatile genetic tool for post-translational control of gene 371 expression in Drosophila melanogaster. Elife 6. doi:10.7554/eLife.30327.

Shanbhag, S. R., Müller, B., and Steinbrecht, R. A. (1999). Atlas of olfactory organs of Drosophila melanogaster 1. Types, external organization, innervation and distribution of olfactory sensilla. Int. $J$. Insect Morphol. Embryol. 28, 377-397. doi:10.1016/S0020-7322(99)00039-2.

Sweeney, S. T., Broadie, K., Keane, J., Niemann, H., and O'Kane, C. J. (1995). Targeted expression of tetanus toxin light chain in Drosophila specifically eliminates synaptic transmission and causes behavioral defects. Neuron 14, 341-51. Available at: http://www.ncbi.nlm.nih.gov/pubmed/7857643 [Accessed December 12, 2018]. 795.e4. doi:10.1016/j.neuron.2017.10.011. 
hs-Flp , tub-GAL80, FRT19A ; 20UAS-6xGFP, UAS-RedStinger; nsyb-GAL4 tub > STOP > IexA , FRT19A

\section{B}

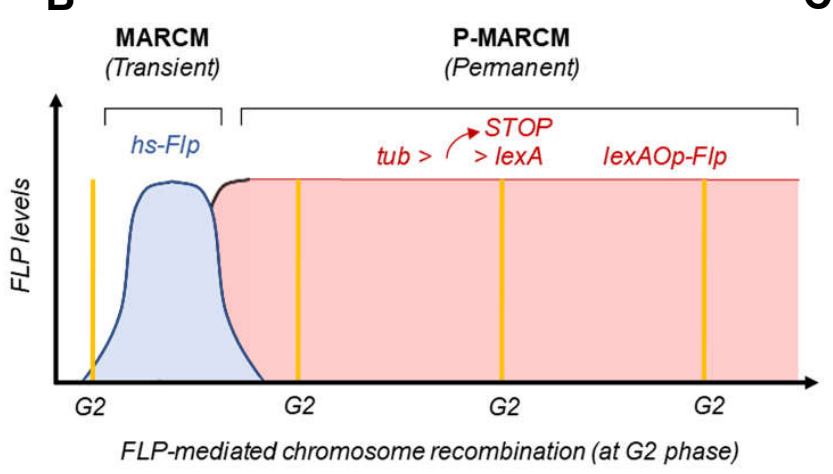

C

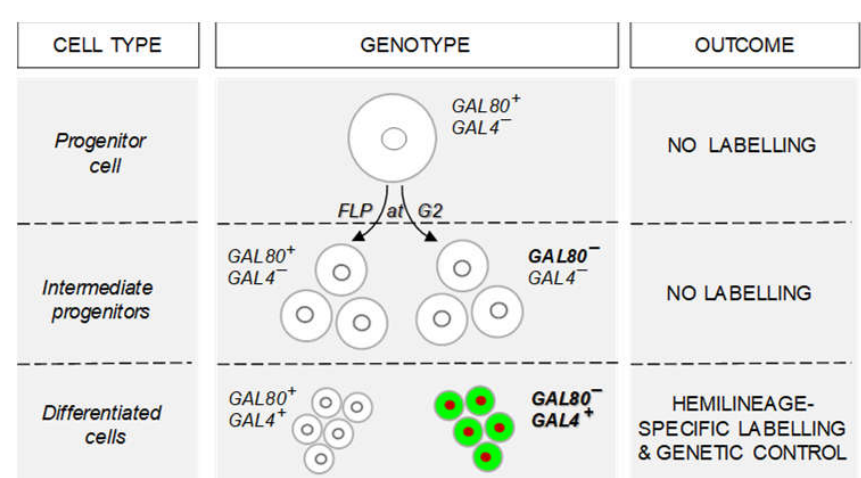

Adult

D Development

\begin{tabular}{|c|c|c|c|c|}
\hline 0 & $17^{\circ} \mathrm{C}$ & 28 & 39 & 5 \\
\hline Cross & & $0 w(H S)$ & $1 w$ & $2 w$ \\
\hline
\end{tabular}

E
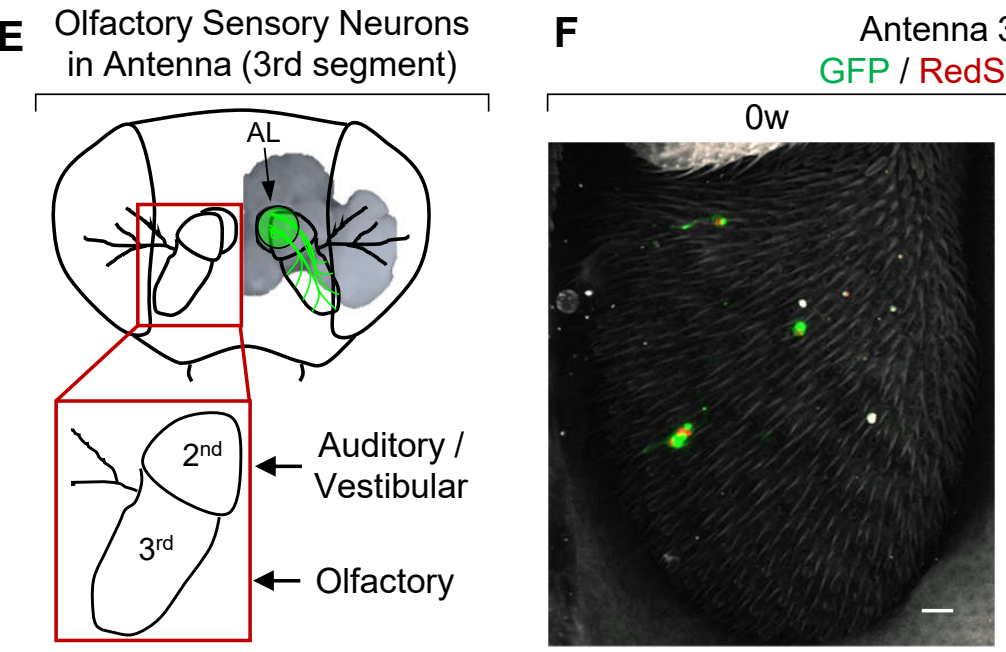

Antenna $3^{\text {rd }}$ Segment
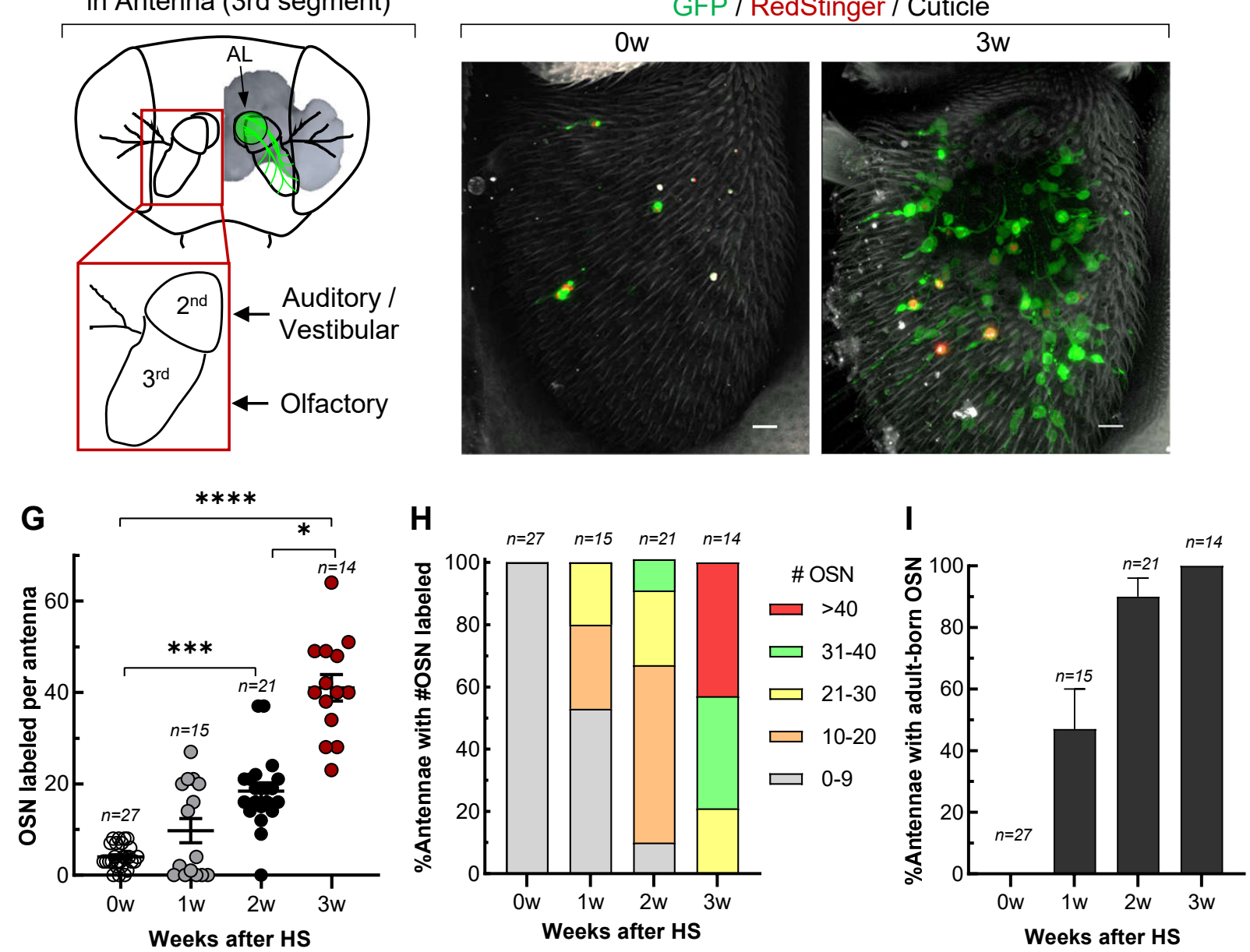

Figure 1. Generation of Olfactory Sensory Neurons (OSN) in the antennae of adult Drosophila. 


\section{Figure 1. Generation of Olfactory Sensory Neurons (OSN) in the antennae of adult Drosophila.}

(A) P-MARCM is a mitotic-dependent lineage tracing system to capture adult-born neurons in Drosophila.

(B) Sustained levels of the recombinase Flippase are expressed through the lexA - lexA-Op system in P-MARCM to allow mitotic recombination even in slowly dividing cells.

(C) Incorporation of cell type-specific GAL4 lines into P-MARCM drives expression of cytoplasmic GFP and nuclear RFP in the cells of interest in the lineage. Additional UAS constructs allow genetic manipulation of adult-born neurons.

(D) Experimental protocol to capture adult-born OSN: P-MARCM flies were kept at $17^{\circ} \mathrm{C}$ during development to minimize background labeling. 3-5 days-old flies were heat shocked to activate P-MARCM and antennae were dissected at 1, 2, or 3 weeks later for confocal imaging.

(E) OSN located on the third antennal segment project axons through the second antennal segment to the Antennal Lobes (AL) in the brain.

(F) P-MARCM reveals new OSN generated over $3 \mathrm{w}$ in the antennae of adult Drosophila. Scale bar: $10 \mathrm{~mm}$.

(G) Sustained generation of OSN over 3 weeks occurs in the antennae of adult flies. ${ }^{*} p<0.05$, $* * * p<0.001, * * * * p<0.0001$. Error bars represent SEM.

(H) Clustering analysis reveals continuous addition of OSN to antennae over time.

(I) The number of antennae with OSN neurogenesis increases over time. By 3 weeks, 100\% of the antennae have generated new OSN. 
A

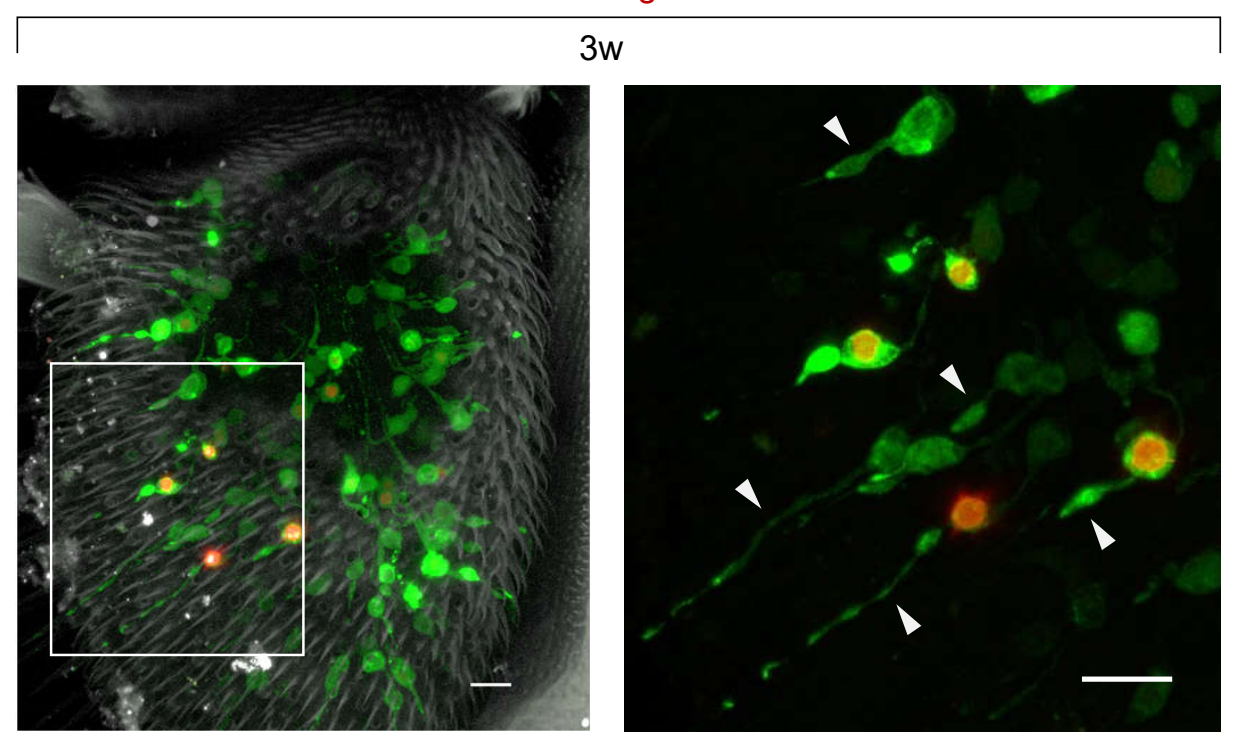

B

Antenna $2^{\text {nd }}$ Segment GFP / RedStinger / Cuticle

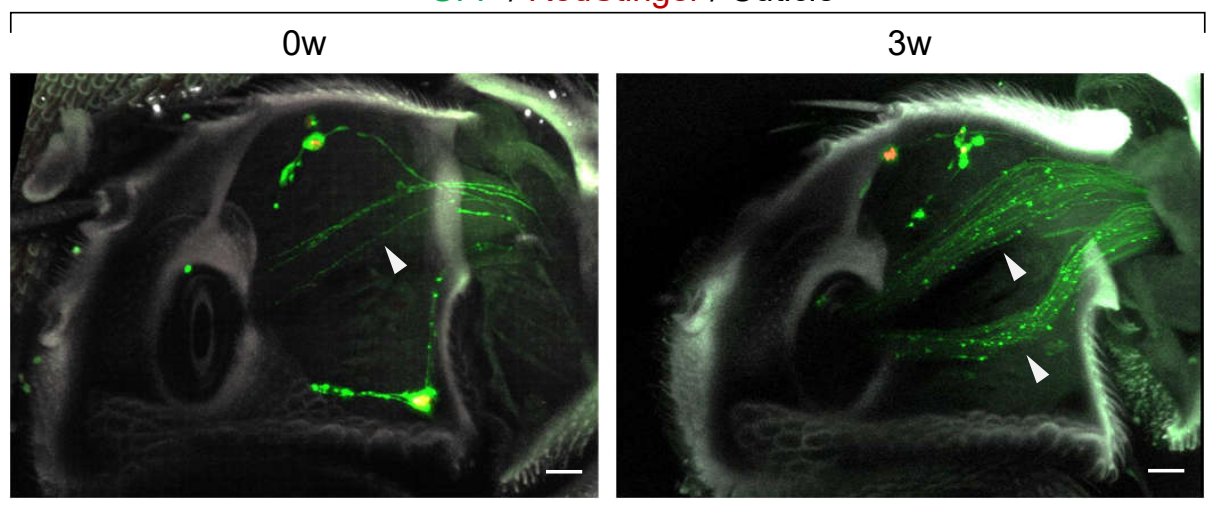

C

Antennal Lobe (Brain)

GFP / nc82

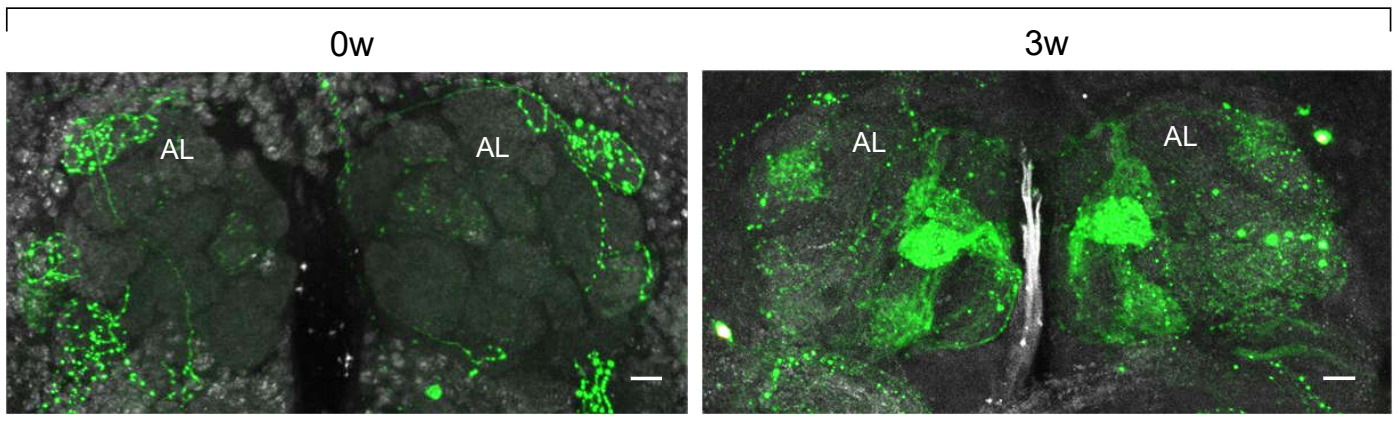

Figure 2. Adult-born OSN develop sensory dendrites and target brain circuitry. 
Figure 2. Adult-born OSN develop sensory dendrites and target brain circuitry.

(A) New OSN develop sensory dendrites of different lengths projecting to sensilla. Magnification panel shows boxed area on left panel.

(B) Newborn OSN in the antennae send axons to the brain through the second antennal segment (arrowheads).

(C) Axons of newborn OSN target different glomeruli in the Antennal Lobes (AL) in the brain. Images presented are for the control $(0 \mathrm{w})$ and experimental $(3 \mathrm{w})$ antennae shown in Figure 1F. Scale bars for all panels: $10 \mu \mathrm{m}$. 

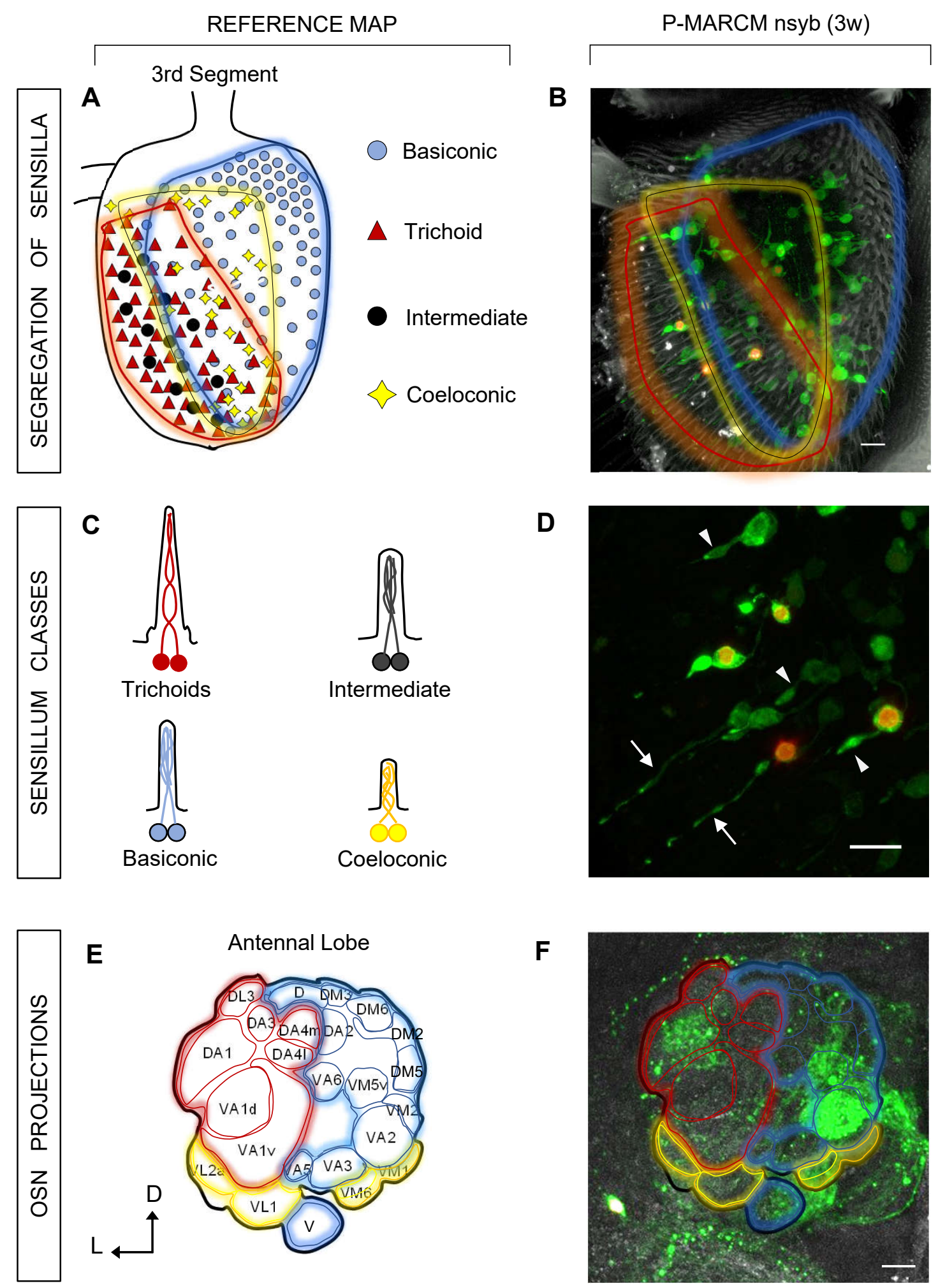

Figure 3. Adult neurogenesis spans to diverse OSN subtypes in the antenna. 


\section{Figure 3. Adult neurogenesis spans to diverse OSN in the antenna.}

(A-B) New OSN are generated in the domains of different sensilla in the antenna. Reference map on panel A based on (Couto et al., 2005; Lin \& Potter, 2015). For simplicity, the three types of Basiconic and Trichoid sensilla are represented as a single one; intermediate and Trichoid sensilla are also both surrounded by the red area.

(C-D) The different lengths of sensory dendrites in newborn OSN are suggestive of neurons in the Trichoid (long, arrows) and Coeloconic (short, arrowheads) sensilla. Cartoons on panel C based on (Couto et al., 2005; Shanbhag et al., 1999). For simplicity, only sensilla containing 2 OSN are represented.

(E-F) Axons of newborn OSN innervate diverse glomeruli in the AL, indicative of neurons expressing diverse olfactory receptors. Reference map on panel E shows glomeruli on the frontal view of AL, based on (Couto et al., 2005). Scale bars for all panels: $10 \mu \mathrm{m}$. 
A

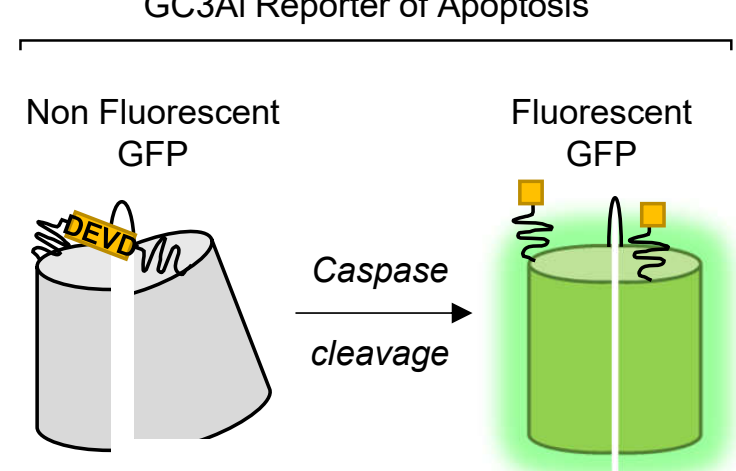

B

nsyb-GAL4, UAS-GC3Ai

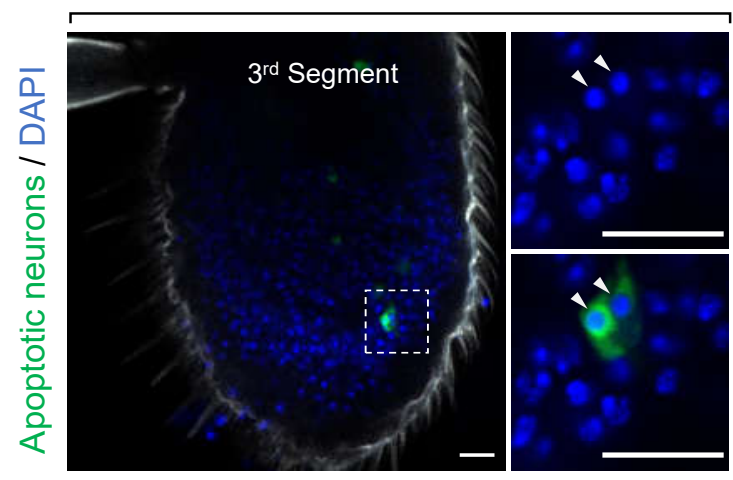

nsyb-GAL4, UAS-GC3Ai

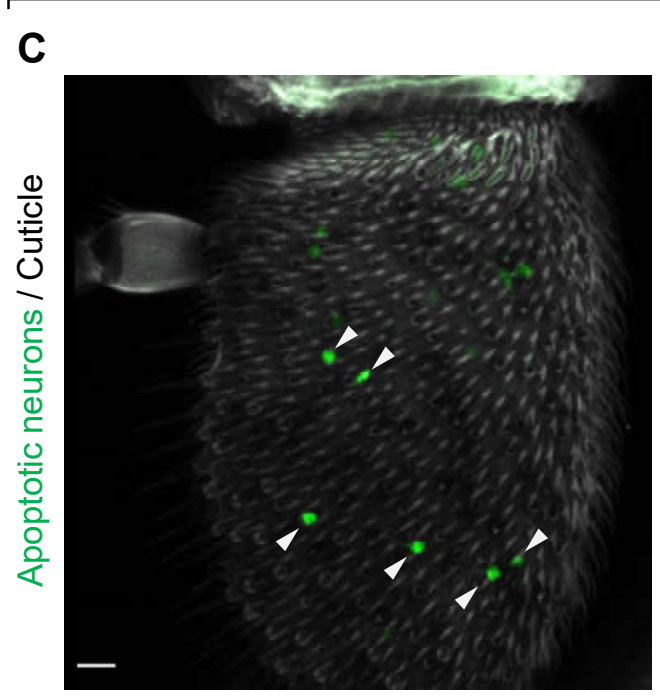

$\mathbf{E}$

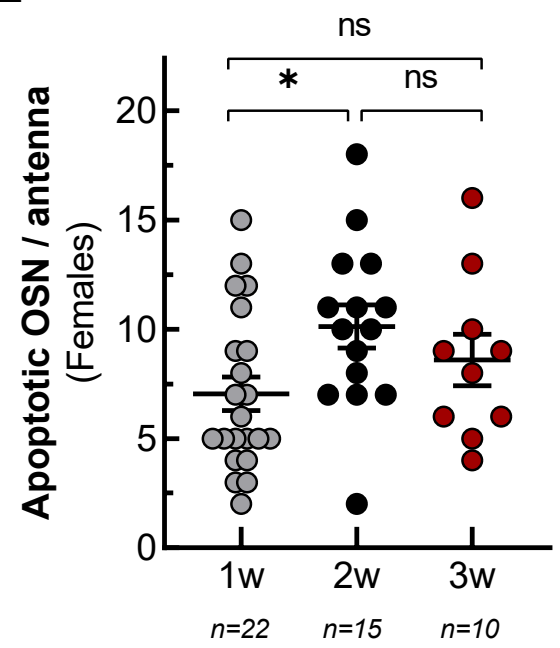

D

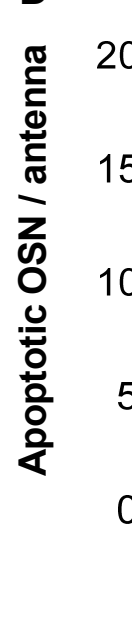

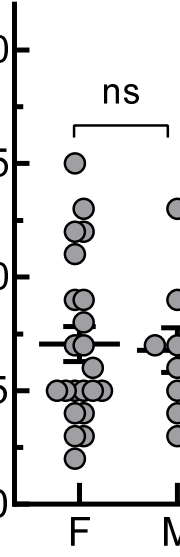

$n=22 \quad n=9$

○ $1 \mathrm{w}$

F

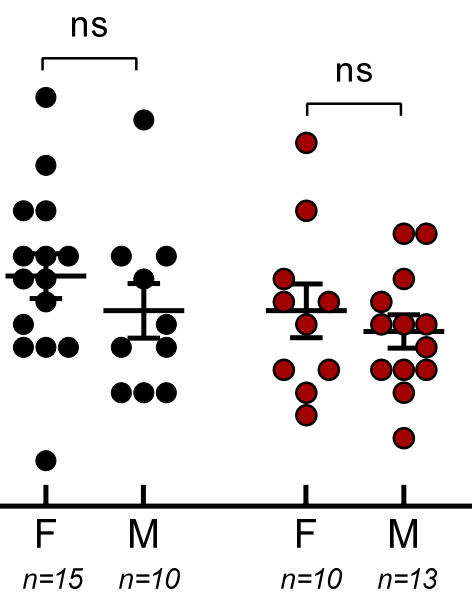

- $2 \mathrm{w}$

- $3 w$
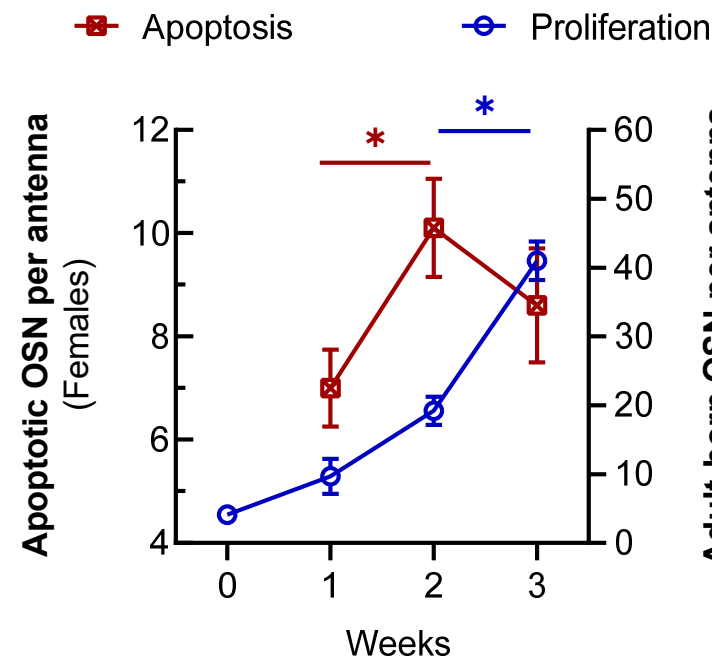

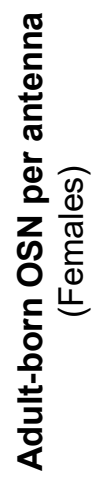

Figure 4. Turnover of adult OSN. 
Figure 4. Turnover of adult OSN.

(A)In the GC3Ai reporter of apoptosis (Schott et al., 2017), an inserted DEVD sequence renders GFP non fluorescent. Upon its cleavage by activated caspases, GFP reconstitutes and becomes fluorescent in apoptotic cells.

(B) GC3Ai is expressed in OSN by nsyb-GAL4 line. Apoptotic OSN labeled by GC3Ai show condensed DNA (arrowheads) as a hallmark of apoptosis.

(C) Apoptotic OSN are detected throughout the $3^{\text {rd }}$ antennal segment by $n s y b>>G C 3 A i$ (arrowheads).

(D) Apoptosis in OSN is consistently detected in Males (M) and Females (F) with no significant difference for each time point analyzed (unpaired t-Test, $p>0.3$ for all cases).

(E) A significant increase in OSN apoptosis is detected in the antennae of female flies from $1 \mathrm{w}$ to $2 \mathrm{w}$ (one-way ANOVA, $* p=0.04$ ).

(F) Physiologic OSN turnover is revealed by a compensatory proliferation following sustained apoptosis of neurons over time. A significant increase in OSN proliferation at $3 \mathrm{w}$ following a significant increase in OSN apoptosis at $2 \mathrm{w}$ further substantiates this interpretation. Scale bars: $10 \mu \mathrm{m}$. Error bars represent SEM. 


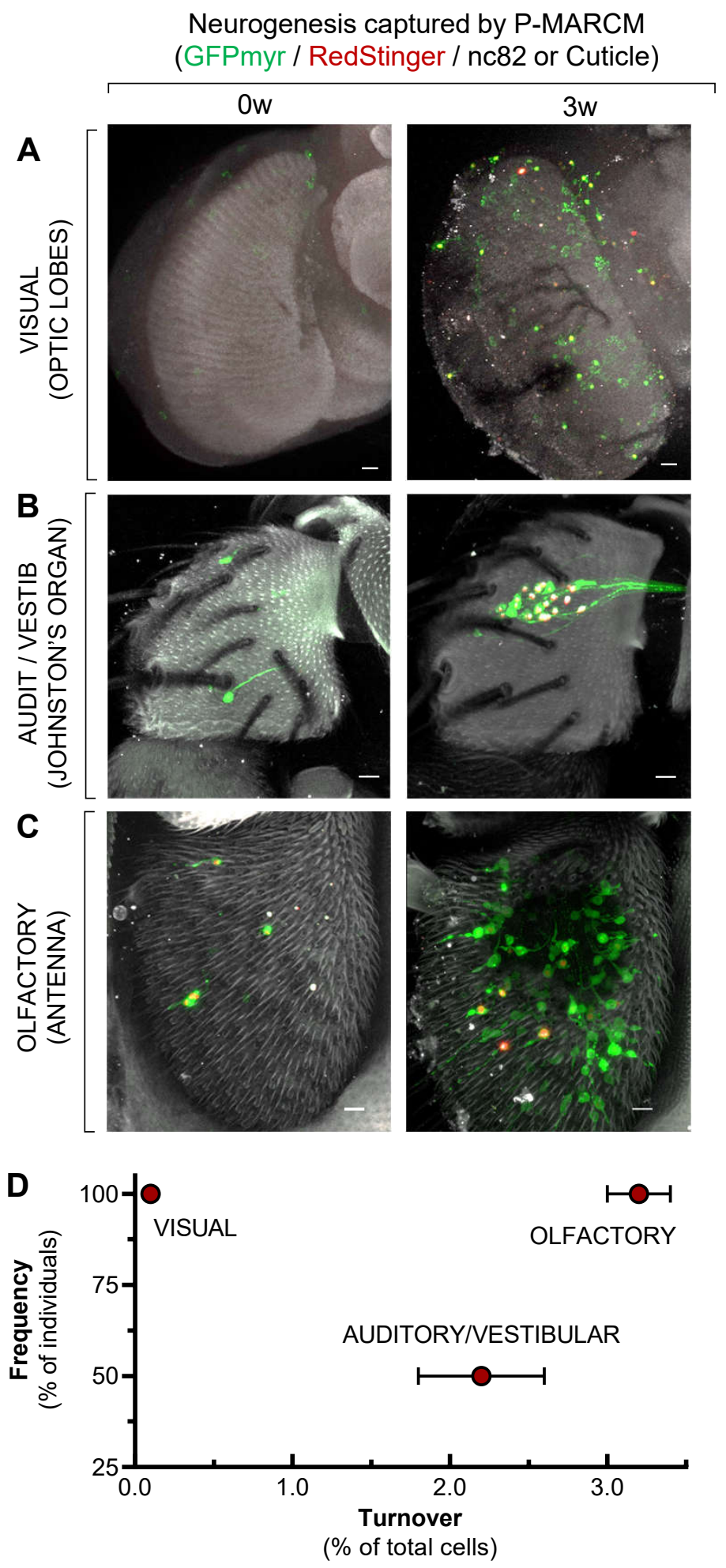

Supplementary Figure 1. Adult neurogenesis accross sensory systems in Drosophila.

(A-C) P-MARCM captures neurogenesis over 3 weeks in the Visual system (Optic Lobes) (A), the Auditory/Vestibular system (JO) (B), and the Olfactory System (antena) (C) of adult Drosophila. For JO, a representative image of neurogenesis at $4 \mathrm{w}$ is shown. Scale bars: $10 \mu \mathrm{m}$.

(D) The Olfactory system shows the highest neurogenesis turnover at $3 \mathrm{w}$ among the three sensory systems analyzed. For auditory/vestibular, an interpolation of $2 \mathrm{w}$ and $4 \mathrm{w}$ data is plotted (Fernández-Hernández et al., 2019). Error bars represent SEM. 